\title{
Characterization of untranslated regions of the salmonid alphavirus 3 (SAV3) genome and construction of a SAV3 based replicon Marius Karlsen*1, Stephane Villoing ${ }^{2}$, Espen Rimstad ${ }^{3}$ and Are Nylund ${ }^{1}$
}

Address: ${ }^{1}$ Department of Biology, University of Bergen, Thor Møhlens gate 55, 5020 Bergen, Norway, 2Intervet Norbio, Thor Møhlens gate 55, 5008 Bergen, Norway and ${ }^{3}$ Norwegian School of Veterinary Science, Oslo, Norway

Email: Marius Karlsen* - marius.karlsen@bio.uib.no; Stephane Villoing - stephane.villoing@sp.intervet.com; Espen Rimstad - espen.rimstad@veths.no; Are Nylund - are.nylund@bio.uib.no

* Corresponding author

Published: 27 October 2009

Virology Journal 2009, 6:173 doi:10.1186/1743-422X-6-173

This article is available from: http://www.virologyj.com/content/6/1/173

(C) 2009 Karlsen et al; licensee BioMed Central Ltd.

This is an Open Access article distributed under the terms of the Creative Commons Attribution License (http://creativecommons.org/licenses/by/2.0), which permits unrestricted use, distribution, and reproduction in any medium, provided the original work is properly cited.
Received: 27 August 2009

Accepted: 27 October 2009

\begin{abstract}
Salmonid alphavirus (SAV) causes disease in farmed salmonid fish and is divided into different genetic subtypes (SAVI-6). Here we report the cloning and characterization of the 5'- and 3'untranslated regions (UTR) of a SAV3 isolated from Atlantic salmon in Norway. The sequences of the UTRs are very similar to those of SAVI and SAV2, but single nucleotide polymorphisms are present, also in the $3^{\prime}$ - conserved sequence element (3'-CSE). Prediction of the RNA secondary structure suggested putative stem-loop structures in both the 5'- and 3'-ends, similar to those of alphaviruses from the terrestrial environment, indicating that the general genome replication initiation strategy for alphaviruses is also utilized by SAV. A DNA replicon vector, pmSAV3, based upon a PVAXI backbone and the SAV3 genome was constructed, and the SAV3 non-structural proteins were used to express a reporter gene controlled by the SAV3 subgenomic promoter. Transfection of pmSAV3 into CHSE and BF2 cell lines resulted in expression of the reporter protein, confirming that the cloned SAV3 replication apparatus and UTRs are functional in fish cells.
\end{abstract}

\section{Findings}

Salmonid alphaviruses (SAVs) cause disease in farmed salmonids both in freshwater and the marine environment in Europe [1]. The virus, also known as Salmon pancreas disease virus, was molecularly characterized during the late 1990-ies, and assigned to the genus Alphavirus in the family Togaviridae [2,3]. Alphaviruses have single-stranded RNA genomes of 11-12 kb length with a 5'-terminal cap and a 3'-terminal polyadenylated tail. The coding sequences are organized into two large non-overlapping open reading frames (ORFs) that are flanked by three untranslated regions (UTRs). The first ORF is approximately $8 \mathrm{~kb}$ and encodes the non-structural proteins (nsPs) 1-4, while the second ORF is approximately $4 \mathrm{~kb}$ and encodes the structural proteins capsid, E3, E2, 6K, TF and E1. The second ORF is transcribed from an anti-sense genome under the control of a subgenomic promoter in the untranslated region that separates the two ORFs $[4,5]$. The genome is replicated by the nsPs, which together with host proteins make up the replicase complex (RC). The nsPs are translated as a polyprotein, P1234, that is cleaved by a papain-like serine protease of the nsP2 component. The different cleavage products of the RC have several roles during replication that include (i) recognition of viral genomic RNA and transcription of an anti-sense genome, (ii) recognition of the anti-sense genome and transcription of a new genome strand and (iii) recognition of the subgenomic promoter on the anti-sense genome and transcription of a subgenomic mRNA that contains the second ORF. The untranslated regions 
(UTRs) in the genomic 5'- and 3'- ends act as promoters for transcription of genomic and anti-genomic RNA. The RNA secondary structure found in conserved sequence elements (CSEs), rather than the primary sequence, appears to be the prominent factor in the function of these promoters [6].

Alphaviruses have been widely used in reverse genetics and protein expression systems. A common strategy used in alphaviral reverse genetics has been cloning of the viral genome under the control of an RNA polymerase promoter following transcription into a capped and polyadenylated self-replicating RNA [7]. In alphavirus-based replicons the subgenomic, second ORF is replaced with that of the gene of interest (GOI). Expression of the GOI is then executed by the alphavirus replication apparatus. Such replicons are frequently used for basic studies of alphavirus replication and for in vivo expression of GOIs, and can be used as vector systems in vaccination. Alphaviral based expression systems are useful for the latter application since they typically provide high expression of the transgene as well as activation of innate antiviral response in the transfected/transducted cell [8].

SAV is not genetically homogenous in Europe. Sequence comparisons of SAV isolates suggest that at least six distinct virus reservoirs exist and this has resulted in evolution into the subtypes SAV1-6 [9-11]. The coding sequence of nsP3 is particularly variable between the subtypes and contains several insertions/deletions with unknown effect in the C-terminal region. The SAV2 subtype appears to be widespread in freshwater farmed rainbow trout in continental Europe, whereas subtypes 1, 4, 5 and 6 have been found in Atlantic salmon from overlapping areas off the coast of Ireland, Northern Ireland and Scotland. In Norway a genetically homogeneous subtype, SAV3, is found to infect both Atlantic salmon and rainbow trout on the southwest coast, but has only occasionally been found in northern Norway $[10,12]$.

A replicon allowing viral subgenomic promoter-driven expression of a GOI, as well as a reverse genetics system, has been developed for an attenuated strain of SAV2 [13]. In that system the SAV2 genome was transcribed by either T7 RNA polymerase or cellular RNA polymerase II, and the system has been useful for functional studies of SAV2 [13]. SAV3 and SAV2 represent two subtypes of the Salmonid alphavirus species showing approximately $7.1 \%$ nucleotide sequence differences in their genomic sequences [10]. SAV3 causes disease in farmed salmonids in the marine grow-out phase, while SAV2 typically causes disease in rainbow trout fingerlings. The optimum temperature for replication may also differ, as it appears to be lower for SAV2 than for SAV3 [1]. In order to learn more about these differences, we sought to obtain tools to study the replication apparatus of SAV3. The genomic ends of SAV3 had not been characterized. Therefore, we cloned and sequenced these from chinook salmon embryo (CHSE) cell cultures infected with SAVH20/03 [10] passage 28, using 5'- and 3'- rapid amplification of cDNA ends (RACE) kits (Invitrogen) as recommended by the manufacturer. Nucleotide sequence alignment of SAV3 UTRs with those of SAV1 and SAV2 (sequences of the UTRs of SAV4-6 subtypes were not available) demonstrated $100 \%$ identity of the 5'-UTR of SAV3 (SAVH20/03) and SAV2 strain rSDV, while four nucleotide polymorphisms were present in the 3 '-UTR. Interestingly, one of these polymorphisms was found in the 3'-CSE (Fig. 1a). The 3'-CSE is conserved among alphaviruses, and functions as promoter for the initiation of minus-strand transcription [6].

The 5'- and 3'-UTRs of SAV are the shortest known among alphaviruses [14]. This has caused speculation as to whether SAV transcription initiation could be independent of a stem-loop structure in the genomic 5'- end [13]. Since secondary structure rather than nucleotide sequence in the 5'-UTR is decisive for initiation of genomic replication in other alphaviruses [6], the lack of a stem-loop in the SAV 5'-UTR would imply a different strategy for polymerase/promoter recognition. The distinct phylogenetic position of SAV, and vast evolutionary distance from terrestrial alphaviruses could make this plausible $[2,15]$. However, in silico analysis using Mfold RNA secondary structure predictor [16] with folding temperature set to $14^{\circ} \mathrm{C}$ (the replication temperature for SAV3), suggested that SAV 5'-UTR might form two short stem-loops in the 5 '-end, and that a portion of the coding sequence of nsP1 is likely to be part of the second structure (Fig. 1b). In the 3'-UTR, four stem loops were predicted (Fig. 1c). Due to the observed polymorphisms in the 3'-CSE, it is predicted that SAV3 has a slightly longer stem loop than SAV2 (Fig. 1d).

Knowledge of the ultimate ends and thus full-length sequence of SAV3 (isolate SAVH20/03), allowed construction of a SAV3 based replicon. Using a similar strategy as previously used for SAV2 [13], we constructed the plasmid pmSAV3 (Fig. 2a). The plasmid has the following characteristics: (i) a pVAX1 (Invitrogen) backbone optimized for DNA-vaccination, with a transcription unit controlled by the cytomegalovirus (CMV) immediate early promoter and a bovine growth hormone (BGH) polyadenylation signal, (ii) the SAV3 genome in which the structural ORF has been replaced with that of the enhanced green fluorescence protein (EGFP), flanked by AgeI and AscI restriction enzyme sites, (iii) a polyadenylated tail fused directly to the 3'-UTR of SAV3, and (iv) a hammerhead ribozyme fused directly to the SAV3 5'-UTR. The latter was included since a correct genomic 5'-end has been reported to be 
A

SAV1 : catctattggtttt-aaaatttcaatac SAV2 : catctattggttttaaaatttcaat-SAV3 : catctattggtttt-aaaatttcaatat

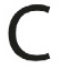

B

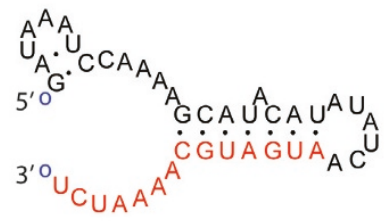

D

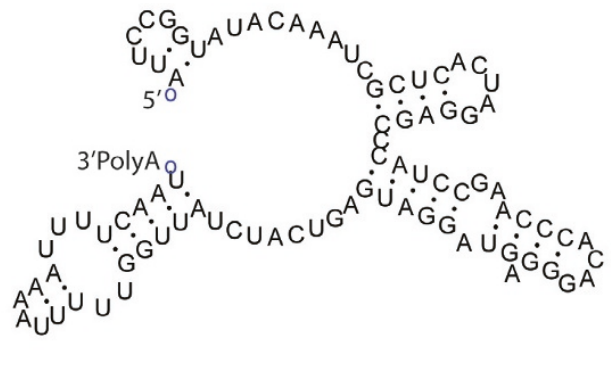

\section{Figure I}

Cloning and characterization of SAV3 5'- and 3'-ends. The 5'- and 3'-ends of SAV3 were cloned and sequenced from the isolate SAVH20/03 and aligned to the 5'- and 3'-UTRs of other SAVs. A) Alignment of the region in the 3'-UTR containing a sequence homologous to the alphavirus $19 \mathrm{nt}$ CSE (underlined). B) In silico RNA secondary structure analysis using Mfold of SAV3 5'-UTR and partial coding sequence. Red letters indicate coding sequence. C) Predicted RNA secondary structures of the 3'-UTRs of SAV2 and SAV3. Position of the polyadenylated tail is indicated.

crucial to obtain efficient replication with SAV2 [9]. Ribozyme generation was done by annealing oligos 5HHribo2/3HHribo2 followed by polymerisation using Klenow fragment (TaKaRa), as earlier described [13]. Primers and restriction enzyme sites that were used for cloning purposes are listed in Table 1 . The authenticity of the plasmid construction was verified by EcoRI, AgeI and AscI (New England Biolabs) digestion (Fig. 2b) and by sequencing as previously described [12]. This information indicated that eight substitutions were present in the RC coding region compared to the nucleotide sequence of passage 20 of the parental strain SAVH20/03 (Table 2). One of the substitutions, the $\mathrm{R}$ to $\mathrm{C}$ in the nsP3 region, was reported to be present in passage 3 of SAVH20/03 [10], suggesting that it could be part of a viral quasispecies.

Transfection of pmSAV3 into CHSE or BF2 cells using Amaxa nucleofector kit T (Lonza) or Metafectene Pro according to producer recommendations resulted in expression of the EGFP reporter that was visualized by flu- orescence microscopy (Fig. 2c). In these experiments transfected cells were kept for $24 \mathrm{~h}$ at $20^{\circ} \mathrm{C}$, then moved to $14^{\circ} \mathrm{C}$, at which SAV3 replication is efficient [12]. The incubation step at $20^{\circ} \mathrm{C}$ enables the efficient transcription from the $\mathrm{CMV}$ promoter, which is more active at $20^{\circ} \mathrm{C}$ than at $14^{\circ} \mathrm{C}$ in CHSE cells (personal observation). In this SAV3 replicon system, expression of the EGFP reporter showed slower kinetics compared to the positive control used, i.e. EGFP under the direct control of the CMV promoter as in the plasmid pEGFP-N1 (Clontech), where EGFP typically can be observed as early as 6-12 h post transfection (p.t) (not shown). The earliest SAV3 replicon expression of EGFP was observed 2 days p.t. in CHSE cells and 3 days p.t. in BF2 cells. The number of positive cells peaked between days 6 and 8 p. t. in CHSE cells and around day 14 p.t. in BF2 cells.

In order to obtain efficient replication the polyadenylated tail had to be fused directly to the 3'-UTR of the SAV genome. Versions of pmSAV3 replicon constructs in which polyadenylation was initiated by the BGH signal in 


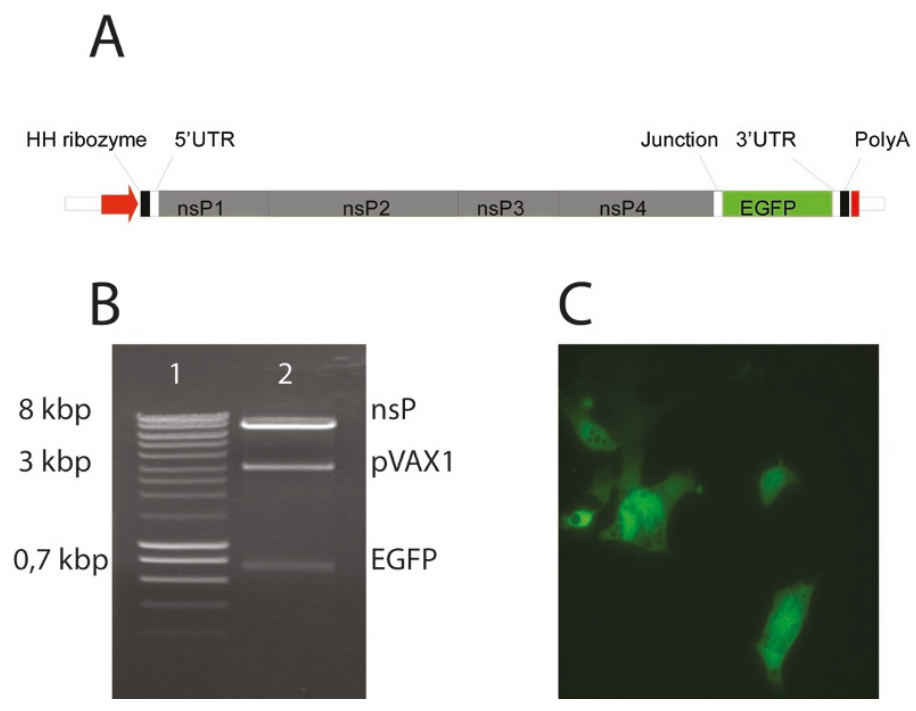

\section{Figure 2}

Construction and evaluation of a SAV3 based replicon. A) A SAV3 replicon is launched from the CMV promoter (red arrow) in the PVAXI backbone, and transcription stops at the BGH polyA signal (red box). A synthetic DNA encoding a hammerhead ribozyme was fused to the SAV3 5'-UTR and a polyadenylated tail was fused to the 3'-UTR. The ORF encoding the SAV3 structural proteins was replaced by an ORF encoding EGFP inserted between introduced Agel and Ascl sites. Position of the EcoRI site used for restriction enzyme analysis is indicated. B) Restriction enzyme analysis by digestion of pmSAV3 with EcoRI, Agel and Ascl. Lane I: Smartladder (Eurogentec). Lane 2: pmSAV3 after triple digest with EcoRI, Agel and Ascl. Bands corresponding to the PVAXI backbone, nsP coding sequence and EGFP coding sequence are indicated. C) Expression of EGFP in BF2 cells after transfection with pmSAV3. Both CHSE and BF2 cells facilitated successful expression of the EGFP reporter. EGFP expression became visible from day 2 p.t. in CHSE cells and 3 d.p.t. in BF2 cells.

Table I: Primers used for construction of pmSAV3.

\begin{tabular}{lll}
\hline Primer & Sequence 5'-3' & REN site \\
\hline KPI & CCGAATTCGTTAAATCCAAAAGCATACATATATCAATGATGC & EcoRI \\
KP2 & CCCGGGGCGGCCCCAAGGTCGAGAACTGAGTTG & \\
KP3 & CCCGGGAGGAGTGACGACTACTGCGTGAAGAAG & Xbal \\
KP4 & GGTCTAGAGTATGATGCAGAAAATATTAAGG & Sacl \\
nsP2SaclF & GAGCTCATGACTGCGGCTGCC & Hpal \\
nsP3HpalR & GTTAACCAAGACTTCCTCTTCGGC & Ascl \\
Ascl3UTRF & GGCGCGCCATTCCGGTATATAAA & Ascl \\
AsclGFPR & GGCGCGCCTTACTTGTACAGCTCGTCCATGC & Xbal, Agel \\
XbalAgelKGFPF & TCTAGACCAACCACCGGTGCCACCATGGTGAGCAAG & Sacl, Nhel \\
5HHribo2 & GGGGAGCTCGCTAGCTGGATTTATCCTGATGAGTCCGTGAGGACG & Nael \\
3HHribo2 & AAACTATAGGAAAGGAATTCCTATAGTCGATAAATCCAAAAGC & \\
NotIXbalPolyAR & CCCGCCGGCGGAGGGGTTAGCTGTGAGATTTTGCATCATTGATATATG & Notl, Xbal \\
NotlXbalPolyA3R & CCGCGGGCCGCTCTAGAT 25 ATTGAAAATTTTAAAAACC & Notl, Xbal \\
\hline
\end{tabular}

Restriction enzyme sites that were used during cloning are indicated in the sequence by bold letters. 
Table 2: Mutations in the pmSAV3 coding region compared to the previously published sequence of SAVH20/03 passage 20 (Accession number DQ149204).

\begin{tabular}{llll}
\hline Position in ORF & Protein product & Nucleotide substitution & Amino acid substitution \\
\hline 306 & nsPI & A to G & Silent \\
702 & nsPI & T to C & Silent \\
2219 & nsP2 & C to A & A to D \\
4098 & nsP2 & A to G & Silent \\
5427 & nsP3 & C to T & Silent \\
5788 & nsP3 & T to C & R to C \\
7593 & nsP4 & A to G & Silent \\
7602 & nsP4 & A to G & Silent \\
\hline
\end{tabular}

Positions refer to nucleotide position in the ORF encoding SAV3 non-structural proteins.

the vector backbone were not functional (not shown). This is similar to previous reports for DNA-launched SINV replicons [17], and suggests that SAV expression is sensitive for erroneous sequences between the 3'-UTR and the polyadenylated tail. Presumably, this has a negative effect on minus-strand synthesis as observed for SINV [18]. A different construct, pmSAV3M10, where the SAV3 3'-CSE was exchanged with SAV2 3'-CSE was also tested for its ability to express the reporter. This plasmid was generated by amplifying the 3'-UTR with primers AscI3UTRF and NotIXbaIPolyAR, where the latter primer sequence contained the SAV2 3'-CSE (Table 2). The 3'-UTR of pmSAV3 was then exchanged with the amplification product through AscI/XbaI cleavage and ligation. In transfection studies this construct showed expression kinetics similar to those observed for pmSAV3, confirming that the SAV3 replicase complex is able to recognise the SAV2 3'-CSE during replication. Moreover, this suggests that the polymorphisms observed in the 3'-CSE have little or no impact for SAV replication, and as predicted by in silico analysis, are of minor importance for the RNA secondary structure.

The pmSAV3 based plasmids can become valuable tools for functional studies of the SAV3 that currently is regarded as enzootic on the Norwegian west coast, but they may also be used for development of vectored vaccines for use in cold-water fish. It will be of interest to compare the performance of SAV3- and SAV2-based replicons [13] in such studies.

\section{Competing interests}

The authors declare that they have no competing interests.

\section{Authors' contributions}

MK planned the study, conducted laboratory and bioinformatical work, analysed results and wrote the manuscript. SV contributed to conception and experimental design, and critically revised the manuscript. ER contributed to the paper by helping in establishment of some of the laboratory methods used, discussion throughout the study and reading and contributing to the writing of the manuscript. AN contributed to the design of the project, discussion through the experimental period, and contributed to the writing of the manuscript.

\section{Acknowledgements}

MK and AN are funded by the University of Bergen and the Norwegian Research Council grant I85I88/S40. SV is employed by Intervet/ScheringPlough animal health. ER is funded by Norwegian School of Veterinary Science. The authors are grateful to Dr. Lindsey Moore for language editing and useful comments on the manuscript.

\section{References}

I. McLoughlin MF, Graham DA: Alphavirus infections in salmonids-a review. J Fish Dis 2007, 30:5 I I-3I.

2. Villoing S, Bearzotti M, Chilmonczyk S, Castric J, Bremont M: Rainbow trout Sleeping disease virus is an atypical alphavirus. J Virol 2000, 74: $173-83$.

3. Weston JH, Welsh MD, McLoughlin MF, Todd D: Salmon pancreas disease virus, an alphavirus infecting farmed Atlantic salmon, Salmo salar L. Virology 1999, 256: 188-95.

4. Firth $A E$, Chung BY, Fleeton MN, Atkins JF: Discovery of frameshifting in Alphavirus 6K resolves a 20-year enigma. Virol J 2008, 5: 108.

5. Strauss JH, Strauss EG: The alphaviruses: gene expression, replication, and evolution. Microbiol Rev 1994, 58:49|-562.

6. Kaariainen L, Ahola T: Functions of alphavirus nonstructural proteins in RNA replication. Prog Nucleic Acid Res Mol Biol 2002, 7I:187-222

7. Schlesinger S: Alphavirus expression vectors. Adv Virus Res 2000, 55:565-77.

8. Schlesinger S: Alphavirus vectors: development and potential therapeutic applications. Expert Opin Biol Ther 200I, I:I77-9I.

9. Fringuelli E, Rowley HM, Wilson JC, Hunter R, Rodger H, Graham DA: Phylogenetic analyses and molecular epidemiology of European salmonid alphaviruses (SAV) based on partial E2 and nsP3 gene nucleotide sequences. J Fish Dis 2008, 3 I :8 I I-23.

10. Hodneland K, Bratland A, Christie KE, Endresen C, Nylund A: New subtype of Salmonid alphavirus (SAV), Togaviridae, from Atlantic salmon Salmo salar and rainbow trout Oncorhynchus mykiss in Norway. Dis Aquat Organ 2005, 66: I 13-20.

II. Weston JH, Graham DA, Branson E, Rowley HM, Walker IW, Jewhurst VA, Jewhurst HL, Todd D: Nucleotide sequence variation in salmonid alphaviruses from outbreaks of salmon pancreas disease and sleeping disease. Dis Aquat Organ 2005, 66: 105-I I.

12. Karlsen M, Hodneland K, Endresen C, Nylund A: Genetic stability within the Norwegian subtype of Salmonid alphavirus (family Togaviridae). Arch Virol 2006, I 5 I:86I-74.

13. Moriette C, Leberre M, Lamoureux A, Lai TL, Bremont M: Recovery of a recombinant Salmonid alphavirus fully attenuated and protective for rainbow trout. J Virol 2006, 80:4088-98.

14. Weston J, Villoing S, Bremont M, Castric J, Pfeffer M, Jewhurst V, McLoughlin M, Rodseth O, Christie KE, Koumans J, Todd D: Comparison of two aquatic alphaviruses, Salmon pancreas disease 
virus and Sleeping disease virus, by using genome sequence analysis, monoclonal reactivity, and cross-infection. J Virol 2002, 76:6I55-63.

15. Powers AM, Brault AC, Shirako Y, Strauss EG, Kang W, Strauss JH Weaver SC: Evolutionary relationships and systematics of the alphaviruses. J Virol 2001, 75: I0II8-31.

16. Zuker M: Mfold web server for nucleic acid folding and hybridization prediction. Nucleic Acids Res 2003, 31:3406- I5.

17. Dubensky TW Jr, Driver DA, Polo JM, Belli BA, Latham EM, Ibanez CE, Chada S, Brumm D, Banks TA, Mento SJ, Jolly DJ, Chang SM: Sindbis virus DNA-based expression vectors: utility for in vitro and in vivo gene transfer. J Virol 1996, 70:508-19.

18. Hardy RW: The role of the 3' terminus of the Sindbis virus genome in minus-strand initiation site selection. Virology 2006, 345:520-31.

Publish with Bio Med Central and every scientist can read your work free of charge

"BioMed Central will be the most significant development for disseminating the results of biomedical research in our lifetime. "

Sir Paul Nurse, Cancer Research UK

Your research papers will be:

- available free of charge to the entire biomedical community

- peer reviewed and published immediately upon acceptance

- cited in PubMed and archived on PubMed Central

- yours - you keep the copyright

Submit your manuscript here:

http://www.biomedcentral.com/info/publishing_adv.asp 